ARTICLE

Received 28 Dec 2012 | Accepted 26 Feb 2013 | Published 27 Mar 2013 DOl: 10.1038/ncomms2652

\title{
Controlled charge trapping by molybdenum disulphide and graphene in ultrathin heterostructured memory devices
}

Min Sup Choi ${ }^{1,2, \star}$, Gwan-Hyoung Lee ${ }^{1,3, \star}$, Young-Jun Yu ${ }^{4,5, \star}$, Dae-Yeong Lee ${ }^{1,2}$, Seung Hwan Lee ${ }^{1,2}$, Philip Kim ${ }^{5}$, James Hone ${ }^{3} \&$ Won Jong Yoo ${ }^{1,2}$

Atomically thin two-dimensional materials have emerged as promising candidates for flexible and transparent electronic applications. Here we show non-volatile memory devices, based on field-effect transistors with large hysteresis, consisting entirely of stacked two-dimensional materials. Graphene and molybdenum disulphide were employed as both channel and charge-trapping layers, whereas hexagonal boron nitride was used as a tunnel barrier. In these ultrathin heterostructured memory devices, the atomically thin molybdenum disulphide or graphene-trapping layer stores charge tunnelled through hexagonal boron nitride, serving as a floating gate to control the charge transport in the graphene or molybdenum disulphide channel. By varying the thicknesses of two-dimensional materials and modifying the stacking order, the hysteresis and conductance polarity of the field-effect transistor can be controlled. These devices show high mobility, high on/off current ratio, large memory window and stable retention, providing a promising route towards flexible and transparent memory devices utilizing atomically thin two-dimensional materials.

\footnotetext{
${ }^{1}$ Samsung-SKKU Graphene Center (SSGC), Sungkyunkwan University, 2066 Seobu-ro, Jangan-gu, Suwon, Gyeonggi-do 440-746, Korea. ${ }^{2}$ Department of Nano Science and Technology, SKKU Advanced Institute of Nano-Technology (SAINT), Sungkyunkwan University, 2066 Seobu-ro, Jangan-gu, Suwon, Gyeonggi-do 440-746, Korea. ${ }^{3}$ Department of Mechanical Engineering, Columbia University, 120 th Street, New York, New York 10027, USA. ${ }^{4}$ Electronics and Telecommunications Research Institute (ETRI), 218 Gajeong-ro, Yuseong-gu, Daejeon 305-700, Korea. ${ }^{5}$ Department of Physics, Columbia University, 120th Street, New York, New York 10027, USA. * These authors contributed equally to this work. Correspondence and requests for materials should be addressed to W. J. Y. (email: yoowj@skku.edu) or to J. H. (email: jh2228@columbia.edu).
} 
T he remarkable properties of graphene, such as high carrier mobility, thermal conductivity, mechanical flexibility and optical transparency, make it a highly promising material for future electronics ${ }^{1,2}$. The ongoing development of graphene electronics has been accompanied by increasing interest in other two-dimensional (2D) layered materials with different electronic properties, which can be combined with graphene into other layered heterostructures ${ }^{3-6}$. For instance, insulating hexagonal boron nitride (hBN) has emerged as an excellent substrate for graphene, yielding graphene devices with improved mobility and lower disorder compared with more conventional dielectrics ${ }^{4}$. Furthermore, $\mathrm{hBN}$ is atomically flat and, thus, it can serve as a uniform tunnelling barrier that allows perfectly planar charge injection ${ }^{7}$. Semiconducting molybdenum disulphide $\left(\mathrm{MoS}_{2}\right)$, another 2D material, shows a transition from an indirect band gap of $1.3 \mathrm{eV}$ in the bulk to a direct band gap of $1.8 \mathrm{eV}$ for a monolayer ${ }^{6}$. $\mathrm{MoS}_{2}$ field-effect transistors (FETs) with high mobility $\left(200 \mathrm{~cm}^{2} \mathrm{~V}^{-1} \mathrm{~s}^{-1}\right)$ and an on/off ratio of $\sim 10^{8}$ have also been recently reported ${ }^{5}$. Such excellent electrical properties, high transparency and flexibility, altogether make these $2 \mathrm{D}$ materials perfect candidates for flexible electronics. Moreover, rapid development in the synthesis of $2 \mathrm{D}$ materials is improving prospects for mass production and large-scale integration of $2 \mathrm{D}$ electronic materials $\mathrm{s}^{1,8-12}$. Multi-stacking of atomically thin $2 \mathrm{D}$ materials enables vertical integration and use of conventional lithography process. Recently, heterostructure of $2 \mathrm{D}$ materials produced by multi-stacking process opened up a route to make new types of material platforms for high-performance devices ${ }^{4,13-15}$.

A number of reports have utilized graphene or graphene oxide in non-volatile memory devices, such as an FET channel, a charge-trapping layer or an electrode ${ }^{16-22}$. It was shown that the large hysteresis in the gate characterization curves of graphene FETs (GFETs) can be applied for memory device operation ${ }^{23}$. It was also demonstrated that this hysteresis arises due to trapped charge in the oxide dielectric layer ${ }^{24}$. However, the relatively slow dynamics and poor controllability of the trap density in these graphene memory devices require further improvement for realistic applications. Furthermore, insulator (or tunnel barrier) and channel material with band gap, which should be ultrathin and stable, have been required for flexible and transparent memory applications. In this sense, the $2 \mathrm{D}$ materials, such as $\mathrm{hBN}$ and $\mathrm{MoS}_{2}$, can be great candidates thanks to their superior electrical and mechanical properties ${ }^{4,7,25}$.

Here we demonstrate memory devices fabricated entirely from stacked $2 \mathrm{D}$ materials. We fabricated two types of $2 \mathrm{D}$ heterostructured memory devices, which show a significant hysteresis and memory performance thanks to the charge-trapping characteristics of graphene and $\mathrm{MoS}_{2}$. One of the two types of the memory devices was fabricated with graphene as the FET channel, hBN as the tunnel barrier and $\mathrm{MoS}_{2}$ as the chargetrapping layer (denoted as GBM) and the other with $\mathrm{MoS}_{2}$ as the FET channel, hBN as the tunnel barrier and graphene as the charge trapping layer (denoted as MBG). These ultrathin heterostructured memory devices (thinner than $10 \mathrm{~nm}$ ) consisting of $2 \mathrm{D}$ materials has great potential for further miniaturization, application in low-cost electronics and flexible memory device applications, especially in the future mobile devices requiring embedded memories integrated into multi-functional system-ona-chip.

\section{Results}

Memory characteristics of heterostructured devices. Heterostructured devices were fabricated by stacking $2 \mathrm{D}$ materials, which are prepared by mechanical exfoliation (see Methods and
Supplementary Fig. S1) In the case of GBM devices, to verify the effect of charge trapping in $\mathrm{MoS}_{2}$, the graphene sample was cut into two regions-one region with and the other without $\mathrm{MoS}_{2}$. Figure 1a shows the circuit diagram of a fabricated GBM device. Figure $1 b, c$ shows optical micrographs of GBM and MBG devices. In Fig. $1 \mathrm{~b}$, a graphene/hBN device without $\mathrm{MoS}_{2}$ is denoted as GB. Supplementary Fig. S2 and Supplementary Table S1 summarize the Raman spectra and photoluminescence (PL) of graphene, $\mathrm{hBN}, \mathrm{MoS}_{2}$ layers and thickness of each layer of the devices fabricated for this study.

Figure 2a shows the transfer curve of one device (GBM3), with an hBN layer of $6 \mathrm{~nm}$ thick and a $\mathrm{MoS}_{2}$ layer of $5 \mathrm{~nm}$ thick. The position of the Dirac point, corresponding to the minimum conductivity, shifts by more than $20 \mathrm{~V}$ and exhibits a large hysteresis when the direction of the gate voltage sweep is reversed. This remarkable hysteresis is related to underlying $\mathrm{MoS}_{2}$ layer, as there is no appreciable hysteresis in the transfer curve of GB3, a graphene device on the same graphene sample as GBM3 but without underlying $\mathrm{MoS}_{2}$ layer, as shown in the inset of Fig. 2a. The field-effect mobility of graphene in GBM3 is

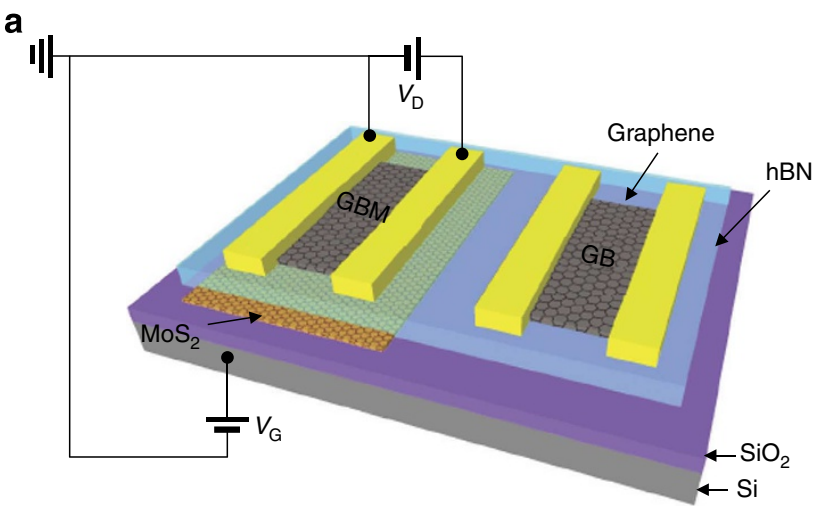

b

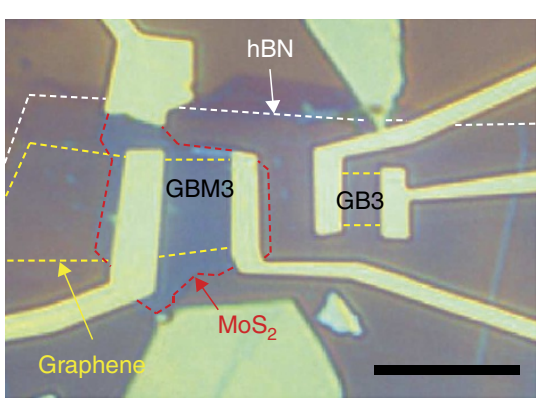

C

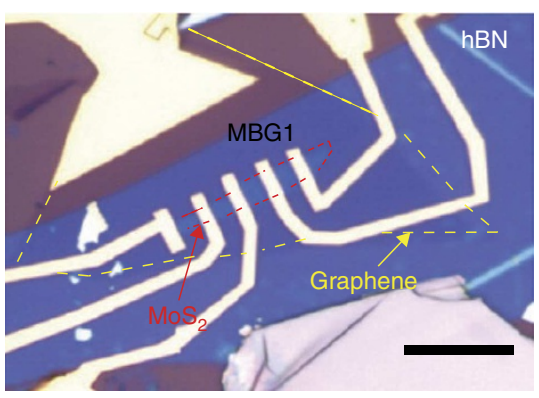

Figure 1 | Structure of heterostructured memory devices. (a) Schematic and circuit diagram of the fabricated device. Optical micrographs of (b) GBM and (c) MBG devices. Scale bars, $10 \mu \mathrm{m}$. The devices of $\mathbf{b}$ and $\mathbf{c}$ are denoted as GBM3, GB3 and MBG1, respectively. The dotted lines indicate the boundaries of each 2D material. 
a

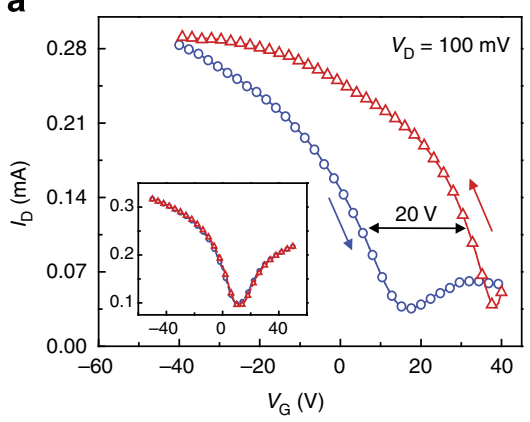

C

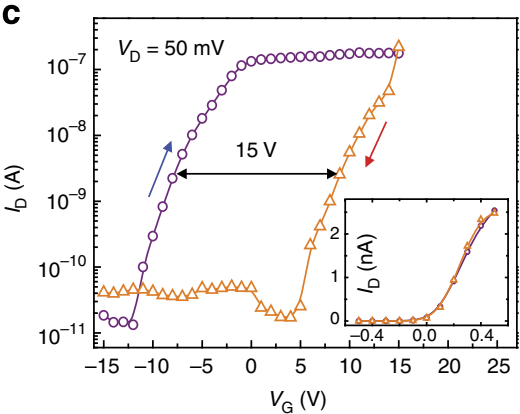

b

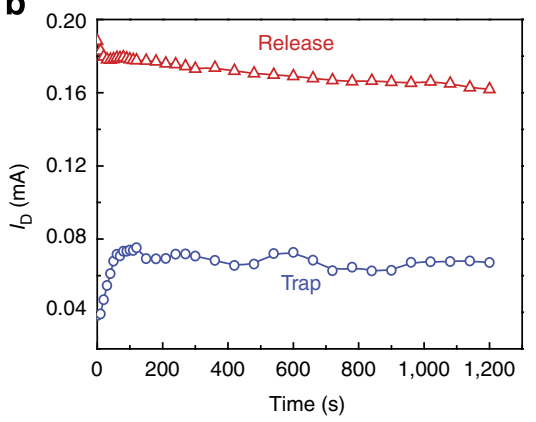

d

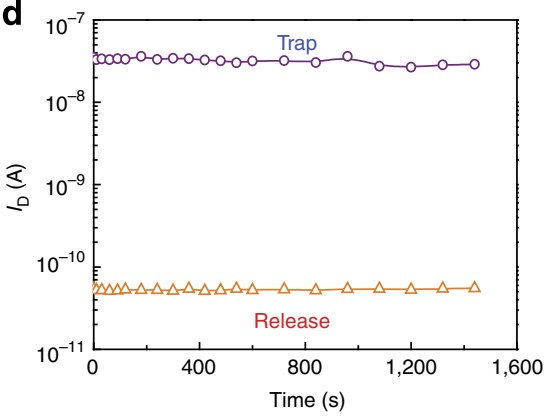

Figure 2 | Transfer and retention characteristics of heterostructured devices. (a) Transfer curve $\left(I_{D}-V_{G}\right)$ and (b) retention performance of the $G B M 3$ device with $\mathrm{hBN}$ of $6 \mathrm{~nm}$ and $\mathrm{MoS}_{2}$ of $5 \mathrm{~nm}$. The inset of a shows a transfer curve of GB3. For GBM devices, all the transfer curves in the main text were obtained at $V_{D}=100 \mathrm{mV}$ and medium sweep rate of $23 \mathrm{Vs}^{-1}$. The retention of $\mathbf{b}$ was measured at $V_{\mathrm{G}}=15 \mathrm{~V}$ with a pulse of $+40 \mathrm{~V}$ (red triangle) and $-40 \mathrm{~V}$ (blue circle), pulse width of $1 \mathrm{~ms}$ and $V_{D}=100 \mathrm{mV}$. (c) Transfer curve and (d) retention performance of the MBG1 device with $\mathrm{hBN}$ of $12 \mathrm{~nm}$, $\mathrm{MoS}_{2}$ of three layers and graphene of two layers. The inset of $\mathbf{c}$ shows a transfer curve of the same device when graphene was used for gating. For MBG devices, all the measurement of transfer curve in the main text was obtained at $V_{D}=50 \mathrm{mV}$ and medium sweep rate of $23 V_{s}{ }^{-1}$. The retention of d was measured at $V_{G}=0 \mathrm{~V}$ with pulse of $-15 \mathrm{~V}$ (violet circle) and $+15 \mathrm{~V}$ (orange triangle), pulse width of $100 \mu \mathrm{s}$ and $V_{D}=50 \mathrm{mV}$.

$4,200 \mathrm{~cm}^{2} \mathrm{~V}^{-1} \mathrm{~s}^{-1}$, which is lower than reported in graphene on $\mathrm{hBN}^{4}$. This mobility degradation is likely due to the presence of a small number of bubbles and wrinkles, as observed in the atomic force microscopy images of Supplementary Fig. S3, which can introduce charge inhomogeneity ${ }^{26}$. However, as hysteresis is not observed in all the GB devices, it is obvious that the existence of underlying $\mathrm{MoS}_{2}$ layer gives rise to the hysteresis in transfer curve of the GBM devices. Even when different voltage sweep rates are applied, no appreciable differences are noted in transfer curves of GBM devices as shown in Supplementary Fig. S4. This indicates that the hysteresis of the transfer curve is not caused by the captured molecules, such as a thin layer of water, at the interface of graphene/hBN ${ }^{23}$.

The observed large gate hysteresis can be utilized for a nonvolatile memory-device operation employing the $\mathrm{MoS}_{2}$ layer as a floating gate. The retention of trapped charge by the $\mathrm{MoS}_{2}$ layer is shown in Fig. 2b. The current $\left(I_{\mathrm{D}}\right)$ in graphene was measured at $V_{\mathrm{G}}=15 \mathrm{~V}$ when gate voltages of $\pm 40 \mathrm{~V}$ with pulse width of $1 \mathrm{~ms}$ were applied. Two states with different currents can be defined as 'trap' and 'release' states. The retention performance shows that trapped charge in the $\mathrm{MoS}_{2}$ layer is maintained without loss of charge. Although $I_{\text {release }} / I_{\text {trap }}$ is low $(\sim 2)$, most of the GBM devices measured in this work exhibit similar retention characteristics regardless of thicknesses of $\mathrm{MoS}_{2}$ charge-trap layer and hBN tunnelling barrier. This charge trapping can be preserved over 100 cycles as shown in the endurance characteristic (Supplementary Fig. S5a). We observed that even GBM devices with monolayer $\mathrm{MoS}_{2}$ exhibit charge-trapping characteristics, resulting in large hysteresis and a good trapped charge-retention property (Supplementary Fig. S6a,b). Most memory devices relying on charge trapping suffer from problems related to charge retention, such as loss of charge by back-tunnelling, injection of carriers of the opposite type or redistribution of charge in defects ${ }^{27}$. In our multi-stack devices, however, the unique device geometry utilizing a high tunnelling barrier and defect-free crystallinity of $2 \mathrm{D}$ crystals provides a solution to circumvent these technical issues.

As single-layer graphene has zero band gap, GFETs have an intrinsically small on/off ratio. To overcome this in FET devices, novel design concepts such as graphene barristor have been proposed $^{28}$. For memory devices, a more reasonable solution is the use of $2 \mathrm{D}$ material with large band gap. Therefore, we fabricated devices with a reversed stacking order, in which single or multilayer $\mathrm{MoS}_{2}$ was employed as a channel and graphene was employed as the charge-trapping layer. The transfer curve of the reverse structure MBG1 device exhibited large hysteresis of $\Delta V \sim 15 \mathrm{~V}$ and high on/off current ratio of $10^{4}$ as shown in Fig. 2c. Similar to GBM devices, different voltage sweep rates showed no appreciable changes in transfer curves of MBG devices as shown in Supplementary Fig. S4d. When graphene was used as a gate electrode, the transfer curve of MBG1 device showed no hysteresis as shown in the inset of Fig. 2c. Moreover, when graphene is grounded, hysteresis of MBG1 device disappeared because of release of the trapped charge from graphene (Supplementary Fig. S7b,d). These results demonstrate that the hysteresis of the MBG device is due to charge trapped in the graphene layer rather than at interfaces between the layers. As a result, good retention and endurance were observed (Fig. $2 \mathrm{~d}$ and Supplementary Fig. S5b). High on/off ratio was maintained over $1,400 \mathrm{~s}$. The carrier mobility of $\mathrm{MoS}_{2}$ in MBG devices ranged from 10 to $40 \mathrm{~cm}^{2} \mathrm{~V}^{-1} \mathrm{~s}^{-1}$, depending on the thickness of $\mathrm{MoS}_{2}$. Even though the carrier mobility of $\mathrm{MoS}_{2}$ is smaller than that of graphene, MBG heterostructure devices should be useful for flexible memory applications requiring the high on/off current 
ratio. As organic memories are being studied as leading contending devices for future flexible device application, we compared the performances of our GBM and MBG to those of reported organic memories (Supplementary Table S2) ${ }^{29,30}$. Compared with organic memories, the heterostructured memory devices fabricated in our study exhibited better performances with advantages of miniaturization, carrier mobility, on/off current ratio, retention and power saving. In addition, the heterostructure memory devices also showed superior stability at high temperature (Supplementary Figs S8 and S9), which implies that these devices can be used in harsh conditions.

Effect of charge-trapping layers. Figure 3 shows proposed energy band diagrams of the tested devices in the flat band state (Fig. 3a, with no contact between layers and no applied bias) and in the carrier transfer state (Fig. 3b,c). The work function and electron affinity of $\mathrm{MoS}_{2}$ are $4.6-4.9 \mathrm{eV}$ and $4.2 \mathrm{eV}$, respectively ${ }^{31,32}$. $\mathrm{hBN}$ has larger band gap $(5.2-5.9 \mathrm{eV})$ and smaller electron affinity $(2-2.3 \mathrm{eV})^{7,33}$. Thererefore, the barrier heights for electron and hole tunnelling through $\mathrm{hBN}\left(\Phi_{\mathrm{e}}\right.$ and $\left.\Phi_{\mathrm{h}}\right)$ are $2.3-2.6 \mathrm{eV}$ and $2.7-3.4 \mathrm{eV}$, respectively. The work function of a charge-neutral monolayer graphene is $4.6 \mathrm{eV}$, and it can be further tuned by external electric field ${ }^{34}$. In the case of GBM, when graphene is influenced by electrical field, carrier density in graphene can be calculated by $n=C_{\text {ox }} / e\left(V_{\mathrm{G}}-V_{0}\right)$, where $C_{\mathrm{ox}}$ and $e$ are capacitance of oxide layer and charge of electron, and $V_{0}$ is the gate voltage corresponding to the charge neutrality point. The shift of Fermi level can then be expressed by $E_{\mathrm{F}}=\operatorname{sgn}(n) \hbar v_{F} \sqrt{\pi|n|}$, where, $v_{F}$ and $\hbar$ are Fermi velocity and Planck's constant, respectively. With $\mathrm{SiO}_{2}$ thickness of $280 \mathrm{~nm}$ and hBN thickness of $10 \mathrm{~nm}, C_{\mathrm{ox}} / e \sim 7.5 \times 10^{10} \mathrm{~cm}^{-2} \mathrm{~V}^{-1}$ is obtained. For the gate voltages of $-40 \mathrm{~V}$ and $+40 \mathrm{~V}$ in GBM3, the calculated Fermi level shifts of single-layer grapheme (SLG) $\left(E_{\mathrm{F}}\right)$ are $-0.19 \mathrm{eV}$ and $+0.22 \mathrm{eV}$, respectively. For this, proximity in energy to conduction $\left(e V_{\mathrm{p}}\right)$ or valence band $\left(e V_{\mathrm{n}}\right)$ of $\mathrm{MoS}_{2}$ should be considered, because tunnelling probability increases with a line-up of energy states in $\mathrm{MoS}_{2}$ and graphene. Although these Fermi level shifts can lower the barrier height for tunnelling (Fig. 3b), the resulting tunnel barrier height is still too high for Schottky thermal emission. Therefore, it is inferred that the main mechanism of charge transfer through $\mathrm{hBN}$ is via quantum tunnelling, which can occur in oxide layers thinner than $6 \mathrm{~nm}^{27}$ (Supplementary Fig. S10). At small gate voltage, the tunnelling current exponentially increases with decreasing hBN thickness, leading to increasing charge injection for trapping ${ }^{7}$. This trend is further supported by the experimental observation that the hysteresis of the transfer characteristic curves in GBM and MBG is larger for thinner hBN barriers (Supplementary Figs. S4 and S11). Moreover, the change in Fermi level $\left(E_{\mathrm{F}}\right)$ in graphene alters the shape of barrier more significantly for a thinner tunnelling barrier, increasing tunnelling current through thinner $\mathrm{hBN}^{13}$. We also note that the tunnelling injection of carriers can be unipolar as indicated in Fig. 3b, electrons in n-doped graphene can be transferred to $\mathrm{MoS}_{2}$ for $V_{\mathrm{G}}>0$, while holes in $\mathrm{p}$-doped graphene can hardly move to $\mathrm{MoS}_{2}$ for $V_{\mathrm{G}}<0$. The electron tunnelling behaviour through $\mathrm{hBN}$ will be explained in detail later. Although carrier transport in the floating gate $\mathrm{MoS}_{2}$ cannot be measured directly, it is considered that the transferred electrons into $\mathrm{MoS}_{2}$ are mobile and stay stably in the conduction band, as the grounded charge-trapping layer of $\mathrm{MoS}_{2}$ causes no hysteresis (Supplementary Fig. S7c), and characteristics of programming and erasing are reproducible as shown in Figs 2 and 4. In MBG devices, graphene acts as a floating gate, which can trap or eject electrons depending on gate voltage as shown in Fig. 3c. When gate voltage is positive in a trapping process, electrons in $\mathrm{MoS}_{2}$ can be pulled and transferred to graphene by tunnelling. The transferred electrons (orange color) can be trapped in graphene. When gate voltage becomes negative in a releasing process, the trapped electrons can be transferred to $\mathrm{MoS}_{2}$, because the negative gate voltage pushes electrons. The Fermi level of graphene is higher than Dirac point in the initial stage of releasing process, because the trapped electrons occupy the energy states over the Dirac point. As the trapped electrons keep transferring to $\mathrm{MoS}_{2}$, the Fermi level of graphene will decrease quickly and become close to the Dirac point. Then, electron-hole recombination probably occurs, resulting in fast removal of the trapped electrons. This also can be supported by the results that trapped charges are released and give rise to no hysteresis when the floating gates are grounded (Supplementary Fig. S7d).

Charging the floated $\mathrm{MoS}_{2}$ gate changes the transfer characteristic of the GBM memories significantly. In particular,
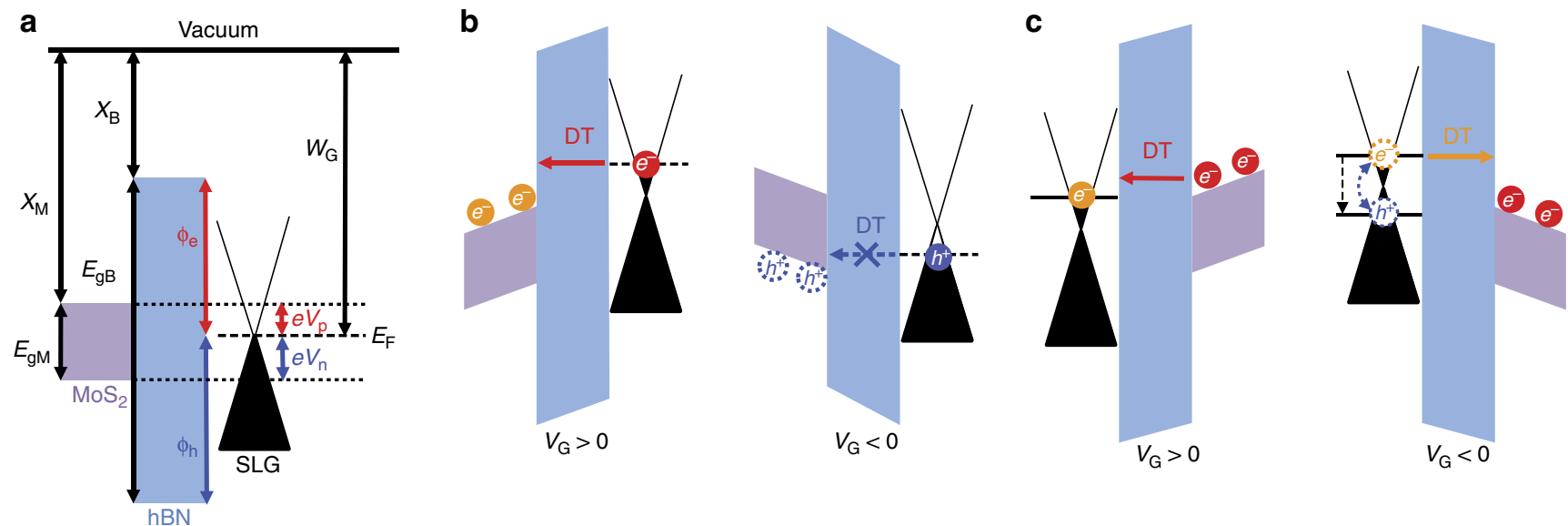

Figure 3 | Energy band diagram of heterostructured memory devices. Energy band diagrams of (a) flat band state ( $\chi$ : electron affinity, $E_{g}$ : band gap, W: work function, $\Phi$ : tunnelling barrier and $e V_{p}\left(e V_{n}\right)$ : difference in energy between conduction band (or valence band) of MoS $S_{2}$ and Fermi level of graphene; $M, B, G$, e and $h$ indicate $\mathrm{MoS}_{2}, \mathrm{hBN}$, graphene, electron and hole, respectively) and (b) carrier transfer state of GBM device. When $V_{G}>0$ is applied, electrons can be transferred from graphene to $\mathrm{MoS}_{2}$ by tunnelling through the $\mathrm{hBN}$ barrier. On the other hand, holes in graphene cannot be transferred to $\mathrm{MoS}_{2}$ at $V_{\mathrm{G}}<0$. (c) Energy band diagrams of carrier transfer state of MBG device. In trapping process of $V_{G}>0$, electrons can be trapped in graphene by tunnelling from $\mathrm{MoS}_{2}$. In releasing process of $V_{G}<0$, the trapped electrons in graphene move back to $\mathrm{MoS}_{2}$ at initial stage. As the Fermi level of graphene is further reduced and below Dirac point, the trapped electrons can recombine with holes generated in graphene. 

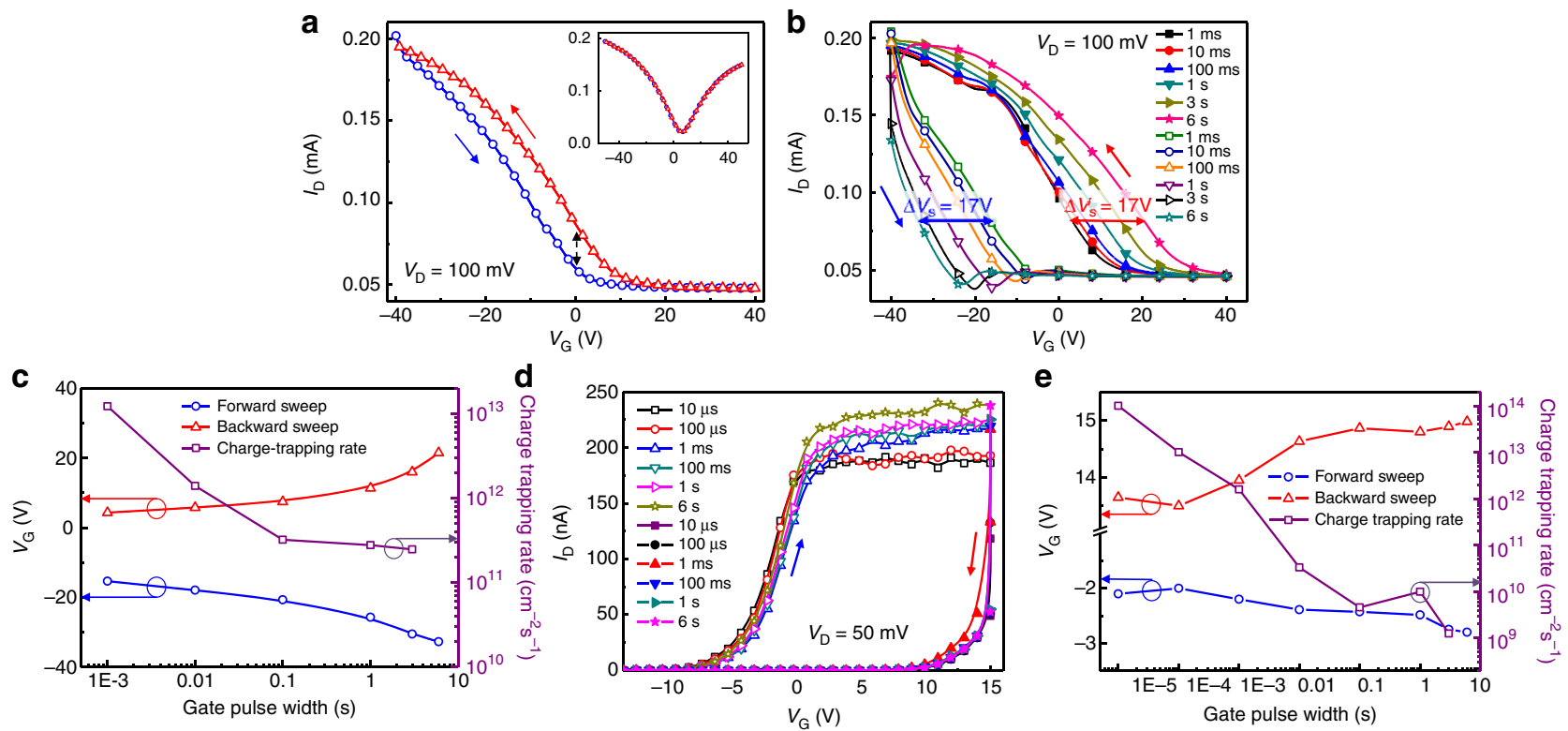

Figure 4 | Effect of pulse width on transfer characteristics and calculation of charge-trapping rate. (a) Transfer curve of GBM2, which has hBN of $11 \mathrm{~nm}$ and $\mathrm{MoS}_{2}$ of $3.3 \mathrm{~nm}$. The inset of a shows the transfer curve of GB2 in the same sample. (b) Transfer curves of GBM2 measured with various pulse widths of $V_{G}= \pm 80 \mathrm{~V}$. The filled and blank symbols represent the values measured with $+80 \mathrm{~V}$ and $-80 \mathrm{~V}$, respectively. (c) Gate voltages, which show the same $I_{D}$ of $78 \mathrm{nA}$, and calculated charge-trapping rate of $\mathrm{GBM} 2$ as a function of gate pulse width. The charge-trapping rate (d $N_{\text {trap }} / \mathrm{dt}$ ) is estimated by gate shift $\Delta V_{S}$ and the pulse width $\Delta t$. (d) Transfer curves of MBG1 measured with various pulse widths of $V_{G}= \pm 30 \mathrm{~V}$. The filled and blank symbols represent the values measured with $+30 \mathrm{~V}$ and $-30 \mathrm{~V}$, respectively. (e) Gate voltages, which show the same $I_{\mathrm{D}}$ of $50 \mathrm{nA}$, and calculated charge-trapping rate of MBG1 as a function of gate pulse width.

when a thick $\mathrm{MoS}_{2}$ layer is used (GBM2), the GFET exhibits a unipolar p-type behaviour, as shown in Fig. 4a. Similar unipolar behaviour was reported in graphene covered with water layer or in doped graphene by metallic oxide, and graphene dual dielectric memory $23,35,36$. To verify that the unipolar behaviour is due to charge trapping in the $\mathrm{MoS}_{2}$ layer, we employed two devices fabricated on a same graphene flake without $\mathrm{MoS}_{2}$-trapping layer, but one on hBN (GB2) and the other on $\mathrm{SiO}_{2}$ (GS2) (Supplementary Fig. S12a). As seen in inset of Fig. 4a, ambipolar behaviour without hysteresis was observed in GB2 (device without $\mathrm{MoS}_{2}$ layer). On the other hand, GS2 (device without hBN substrate nor $\mathrm{MoS}_{2}$ ) exhibits moderate hysteresis (shift less than $5 \mathrm{~V}$ ), presumably due to adsorbed molecules and charge impurities in $\mathrm{SiO}_{2}$ (ref. 23), but the behaviour remains strongly ambipolar (Supplementary Fig. S12b), indicating that the amount of trapped charge is not significant compared with the $\mathrm{MoS}_{2}$ floating gate. For the thick hBN barrier of $11 \mathrm{~nm}$, the memory operation is also poor. As shown in Fig. 4b, although the hysteresis in p-type GFET operation can be utilized for memory operation, the on/off current ratio was smaller than that obtained using more optimized heterostacks demonstrated in Fig. 2 (GBM3). Similarly, the thicknesses of $\mathrm{hBN}$ and graphene in MBG devices influence the FET performance (see Supplementary Fig. S11). When the thicknesses of the tunnel barrier of hBN and charge-trapping layer of graphene are reduced, larger hysteresis and higher on/off current ratio are observed. This is because larger amount of charge can tunnel through thinner hBN and the electric field by a gate voltage is more weakly screened by thinner graphene $e^{7,13,37}$.

\section{Discussion}

We now discuss a potential source of the asymmetric gate characteristics with the $\mathrm{MoS}_{2}$ floating gate. The asymmetry of the observed hysteresis suggests that tunnelling of electrons and holes at positive and negative gate voltages is asymmetric. This asymmetry can be due to different tunnel barrier heights for electrons and holes ${ }^{13}$, or a larger effective mass of holes $\left(m_{\mathrm{h}}=0.5 m_{0}\right)$ than electrons $\left(m_{\mathrm{e}}=0.26 m_{0}\right)$ in $\mathrm{hBN}^{38}$. A large electron tunnelling probability ${ }^{39}$ can lead to a gate-independent channel current at positive gate voltages, as shown in GBM2 and GBM3; electrons in the graphene tunnel rapidly into the $\mathrm{MoS}_{2}$ trap layer and then screen the electric field from the back gate to reach the graphene channel, whereas the low possibility of holetunnelling process does not provide enough charge for screening. This effect is not seen in GBM1, with monolayer $\mathrm{MoS}_{2}$ (Supplementary Fig. S4a); in such a device with very thin $\mathrm{MoS}_{2}$-trapping layers, the amount of trapped charges might not be enough to provide sufficient gate-field screening. The larger hysteresis observed in GBM3 than that in GBM2 (Supplementary Fig. S4b,c) is because of the thinner hBN tunnelling layer and thicker $\mathrm{MoS}_{2}$-trapping layer in GBM3, which induces larger electron tunnelling current providing better screening.

We now discuss the dynamic transition rate of floating gate devices. For this purpose, we pulse the gate and measure the transfer characteristics to estimate the trapping and detrapping rates of the charge into and from the charge-trapping layer. Figure $4 \mathrm{~b}$ shows the hysteresis of device GBM2 as a function of gate pulse width. Here the gate pulses of $\pm 80 \mathrm{~V}$ were applied, with the varying pulse width in the range of $1 \mathrm{~ms}-6 \mathrm{~s}$ followed by the transfer curve measurement. The transfer characteristics are measured as reverse sweeps $(+40$ to $-40 \mathrm{~V})$ following $+80 \mathrm{~V}$ gate pulses, and forward sweeps $(-40$ to $+40 \mathrm{~V})$ following $-80 \mathrm{~V}$ gate pulses. The transfer curve hysteresis loop widens with increasing pulse width up to $6 \mathrm{~s}$, and saturates after that (Fig. $4 \mathrm{~b})$. As the saturated gate voltage shift $\left(\Delta V_{\mathrm{s}}\right)$ is $\sim 17 \mathrm{~V}$, charge-trap density is estimated to be $N=C_{\mathrm{ox}} \Delta V_{\mathrm{s}} / e \sim 1.28 \times 10^{12}$ $\mathrm{cm}^{-2}$, which is larger than a typical trap-charge density of 
$2.5 \times 10^{11} \mathrm{~cm}^{-2}$ in $\mathrm{SiO}_{2}$ (ref. 40). The electron trapping rate in $\mathrm{MoS}_{2}$ (or, equivalently, the hole-releasing rate from $\mathrm{MoS}_{2}$ ) is then estimated from $\left(\mathrm{d} N_{\text {trap }} / \mathrm{dt}\right)=\left(C_{\text {ox }} / e\right)\left(\mathrm{d} V_{\mathrm{G}} / \mathrm{d} t\right)$ by using $\mathrm{d} V_{\mathrm{G}} / \mathrm{d} t$ $\sim \Delta V_{\mathrm{s}} / \Delta t$, where $\Delta t$ is the pulse width and $\Delta V_{\mathrm{s}}$ is the gate voltage shift. Figure $4 \mathrm{c}$ shows both $\Delta V_{\mathrm{s}}$ and charge-trapping rate as a function of gate pulse width. The charge-trapping rate $\left(\mathrm{d} N_{\text {trap }} / \mathrm{d} t\right)$ varies from $10^{13}$ to $10^{11} \mathrm{~cm}^{-2} \mathrm{~s}^{-1}$ as the pulse width changes from $1 \mathrm{~ms}$ to $6 \mathrm{~s}$, indicating the energy level of the trap sites may differ considerably. We also note that the charge-trapping rate is relatively fast compared with a typical value, $\sim 10^{9} \mathrm{~cm}^{-2} \mathrm{~s}^{-1}$, in metal-insulator-semiconductor ${ }^{41}$. Separating from the trapping rate, the charge-transfer rate, $\mathrm{d} N_{\text {transfer }} / \mathrm{d} t$, governed by the tunnelling process, can be estimated from the tunnelling current. In principle, the charge-transfer rate can be expressed by $\mathrm{d} N_{\text {transfer }} / \mathrm{d} t=I_{\text {tunnel }} / A e$, where $I_{\text {tunnel }}, A$ and $e$ are tunnel current, device area and electric charge, respectively. Although we have not monitored the gate tunnelling current, we can utilize the tunnelling measurement result across an hBN layer of similar thickness (see Supplementary Fig. S10), where a charge-transfer rate of $1.18 \times 10^{13} \mathrm{~cm}^{-2} \mathrm{~s}^{-1}$ is estimated. Note that this value is similar to the charge-trapping rate for the short period of pulse $<1 \mathrm{~ms}$, but larger for the longer period of pulse shown in Fig. 4c. This suggests that trapping dynamic control is more important than the tunnelling process control for fast memory operation at this point. We believe that this chargetrapping rate can be further enhanced by optimizing the thickness of hBN and $\mathrm{MoS}_{2}$ layers, so that $\Delta V$ can be enlarged to be more suitable for faster memory device application. Figure $4 \mathrm{~d}$ shows transfer curves of MBG1 measured with various pulse widths of $V_{\mathrm{G}}= \pm 30 \mathrm{~V}$. Both $\Delta V_{\mathrm{s}}$ and chargetrapping rate as a function of gate pulse width are shown in Fig. 4e. The MBG device showed the reasonable memory window even at short pulse width of $10 \mu \mathrm{s}$. The charge-trapping rate varies from $10^{14}$ to $10^{9} \mathrm{~cm}^{-2} \mathrm{~s}^{-1}$. It should be noted that chargetrapping rate at the same pulse width is slower compared with GBM devices. As the thickness of hBN used in MBG and GBM devices here is similar, the difference in charge-trapping rate is attributed to the smaller density of states in the MBG device compared with the GBM device.

We studied the charge-trapping characteristics of $\mathrm{MoS}_{2}$ and graphene in multi-stacked graphene/hBN/MoS $(\mathrm{GBM})$ and $\mathrm{MoS}_{2} / \mathrm{hBN} /$ graphene (MBG) devices. GBM devices on the stacked $\mathrm{hBN} / \mathrm{MoS}_{2}$ showed different hysteresis characteristics, depending on the thicknesses of $\mathrm{MoS}_{2}$ and hBN. From the measurement of retention and endurance characteristics, it was confirmed that the $\mathrm{MoS}_{2}$ layer acts as an effective charge-trapping layer. When thicker $\mathrm{MoS}_{2}$ layer and thinner hBN were employed, unipolar conductance and larger hysteresis were observed because of effective electron tunnelling and electric-field screening. Meanwhile, when the reversed stacking structure of MBG was investigated, high on/off current ratio and large memory window were attained. This study provides a promising route of future flexible and transparent memory device operation, utilizing ultrathin and flexible $2 \mathrm{D}$ materials.

\section{Methods}

Device fabrication. Heterostructured devices were fabricated by stacking $2 \mathrm{D}$ materials. For GBM devices, thin layers of $\mathrm{MoS}_{2}$ were mechanically exfoliated on a silicon wafer with 280 -nm-thick $\mathrm{SiO}_{2}$. After hBN and SLG were exfoliated onto wafers coated with polymethyl methacrylate (PMMA) and a thin release layer, PMMA film was removed from the wafer, then hBN and SLG were sequentially transferred onto the wafer containing the $\mathrm{MoS}_{2}$, as reported previously 4,13 (see Supplementary Fig. S1). MBG devices were fabricated by a similar method, in a reverse-stacking order. For removal of PMMA residues generated during the stacking process, the samples were annealed at $345^{\circ} \mathrm{C}$ by flowing $\mathrm{H}_{2} / \mathrm{Ar}$-forming gas between transfer steps ${ }^{4,13}$. Source and drain electrodes were patterned using electron-beam lithography and deposition of $\mathrm{Cr} / \mathrm{Pd} / \mathrm{Au}(1 / 10 / 50 \mathrm{~nm})$ for GBM and $\mathrm{Ti} / \mathrm{Au}(0.5 / 50 \mathrm{~nm})$ for MBG, where the doped Si substrate underneath the $\mathrm{SiO}_{2}$ layer was used as a back gate. To verify the effect of charge-trapping layer, two devices with or without charge-trapping layer are fabricated by an additional electron-beam lithography process followed by oxygen plasma etching.

Characterization of materials. Atomic force microscopy (Park Systems, XE-100) and Raman spectroscopy (Renishaw, inVia) were used to ensure the qualities and thicknesses of graphene, hBN and $\mathrm{MoS}_{2}$. The laser of $532 \mathrm{~nm}$ wavelength was used for the excitation in Raman spectroscopy. Even though Raman spectra of $\mathrm{MoS}_{2}$ covered by hBN are not sharp as shown in Supplementary Fig. S2a, the separation between two peaks indicate the number of layers. To check the quality of $\mathrm{MoS}_{2}$, $\mathrm{hBN}$ and graphene, therefore, the exfoliated flakes were tested using Raman spectroscopy and micro-PL spectroscopy before the transfer steps as shown in Supplementary Fig. S2b-d. It is difficult to estimate the defect density in graphene sitting on $\mathrm{hBN}$, because the $\mathrm{D}$ peak of graphene and the main peak of hBN are located at the same position of $1,370 \mathrm{~cm}^{-1}$ (Supplementary Fig. S2d) ${ }^{42}$. As shown in Supplementary Fig. S2a,b, $\mathrm{MoS}_{2}$ flakes with various thicknesses showed different peak separations and PL intensities as reported ${ }^{6,43}$. The narrow width $(\sim 80 \mathrm{meV})$ of PL peak around $1.84 \mathrm{eV}$ indicates that $\mathrm{MoS}_{2}$ has high crystallinity ${ }^{6}$.

Electrical characterization of devices. Electrical properties of fabricated devices were measured with a semiconductor parameter analyzer (Agilent, 4155C) in vacuum and at room temperature. The fabricated GBM and MBG devices showed high mobilities of graphene $\left(\sim 4,200 \mathrm{~cm}^{2} \mathrm{~V}^{-1} \mathrm{~s}^{-1}\right)$ and $\operatorname{MoS}_{2}\left(\sim 35 \mathrm{~cm}^{2} \mathrm{~V}^{-1} \mathrm{~s}^{-1}\right.$ for three layers). For measurement of tunnelling current through hBN, a simple metal-insulator-metal device was fabricated as shown in Supplementary Fig. S10a. To make a flat bottom electrode, a pristine few-layer graphene was used. The breakdown voltage for Fowler-Nordheim tunnelling in this device is $\sim 6 \mathrm{~V}$ $\left(6 \mathrm{MV} \mathrm{cm}^{-1}\right)$, which corresponds to the electric field of $180 \mathrm{~V}$ when using Si back gate in a $\mathrm{Si}$ substrate with 300 -nm-thick $\mathrm{SiO}_{2}$. This indicates that, because back gate voltage of $40 \mathrm{~V}$ was used in the GBM devices, the Fowler-Nordheim tunnelling does not take place in the devices. Even though the dielectric strength in this device is smaller than the reported value $\left(8 \mathrm{MV} \mathrm{cm}^{-1}\right.$; ref. 7$)$, high-temperature treatment-like annealing process can lower the dielectric strength of hBN by generating defects ${ }^{44}$. As the annealing temperature increases, tunnelling current through hBN was enhanced probably by trap-assisted tunnelling. As shown in Supplementary Fig. S10b, tunnelling current through hBN slightly increases with temperature. However, tunnelling was not affected by exposure of light $(100 \mathrm{~W})$ as shown in Supplementary Fig. S10c.

Measurement of temperature dependence. To clarify the effect of temperature on tunnelling and charge trapping, transfer curves of the GBM4 and MBG3 devices were obtained at different temperature as shown in Supplementary Fig. S8. For GBM4 device, even though a small decrease in on-current, maybe due to thermal disturbance, was observed, large hysteresis was maintained at $200^{\circ} \mathrm{C}$. It is estimated that the larger memory window and two charge-neutral points at both negativeand positive-gate voltages are attributed to p-doping of graphene during the fabrication process. In contrast, the MBG3 device exhibited no significant temperature dependence as shown in Supplementary Fig. S8b. The small increase in hysteresis and on-current are probably due to thermal excitation to overcome the contact barrier between $\mathrm{MoS}_{2}$ and metal electrode. As shown in Supplementary Fig. S9, there is no appreciable difference in retention performance of the MBG3 device even at $200^{\circ} \mathrm{C}$. The trapped charge can be released by back-tunnelling, and tunnelling can be assisted by high temperature. However, our result indicates that the trapped charge can be stored efficiently without any loss. Therefore, it is evident that the heterostructure memory devices suggested in this study are stable at high temperature; therefore, it can be applied in the harsh conditions.

\section{References}

1. Bae, S. et al. Roll-to-roll production of 30-inch graphene films for transparent electrodes. Nat. Nanotechnol. 5, 574-578 (2010).

2. Schwierz, F. Graphene transistors. Nat. Nanotechnol. 5, 487-496 (2010).

3. Novoselov, K. S. et al. Electric field effect in atomically thin carbon films. Science 306, 666-669 (2004).

4. Dean, C. R. et al. Boron nitride substrates for high-quality graphene electronics. Nat. Nanotechnol. 5, 722-726 (2010)

5. Radisavljevic, B., Radenovic, A., Brivio, J., Giacometti, V. \& Kis, A. Single-layer MoS2 transistors. Nat. Nanotechnol. 6, 147-150 (2011).

6. Mak, K. F., Lee, C., Hone, J., Shan, J. \& Heinz, T. F. Atomically thin MoS2: a new direct-gap semiconductor. Phys. Rev. Lett. 105, 136805 (2010).

7. Lee, G. H. et al. Electron tunneling through atomically flat and ultrathin hexagonal boron nitride. Appl. Phys. Lett. 99, 243114 (2011).

8. Li, X. S. et al. Large-area synthesis of high-quality and uniform graphene films on copper foils. Science 324, 1312-1314 (2009)

9. Kim, K. S. et al. Large-scale pattern growth of graphene films for stretchable transparent electrodes. Nature 457, 706-710 (2009)

10. Song, L. et al. Large scale growth and characterization of atomic hexagonal boron nitride layers. Nano Lett. 10, 3209-3215 (2010). 
11. Lee, K. H. et al. Large-scale synthesis of high-quality hexagonal boron nitride nanosheets for large-area graphene electronics. Nano Lett. 12, 714-718 (2012).

12. Zhan, Y., Liu, Z., Najmaei, S., Ajayan, P. M. \& Lou, J. Large-area vapor-phase growth and characterization of MoS2 atomic layers on a SiO2 substrate. Small 8, 966-971 (2012).

13. Britnell, L. et al. Field-effect tunneling transistor based on vertical graphene heterostructures. Science 335, 947-950 (2012).

14. Castro Neto, A. H. \& Novoselov, K. New directions in science and technology: two-dimensional crystals. Rep. Prog. Phys. 74, $082501-082509$ (2011).

15. Novoselov, K. S. \& Castro Neto, A. H. Two-dimensional crystals-based heterostructures: materials with tailored properties. Phys. Script. T146, 014006 (2012).

16. Hong, S. K., Kim, J. E., Kim, S. O., Choi, S. Y. \& Cho, B. J. Flexible resistive switching memory device based on graphene oxide. IEEE Electr. Device. Lett. 31, 1005-1007 (2010).

17. Stutzel, E. U. et al. A graphene nanoribbon memory cell. Small 6, 2822-2825 (2010).

18. Zhan, N., Olmedo, M., Wang, G. P. \& Liu, J. L. Graphene based nickel nanocrystal flash memory. Appl. Phys. Lett. 99, 113112 (2011).

19. Park, J. K., Song, S. M., Mun, J. H. \& Cho, B. J. Graphene gate electrode for MOS structure-based electronic devices. Nano Lett. 11, 5383-5386 (2011).

20. Hong, A. J. et al. Graphene flash memory. ACS Nano 5, 7812-7817 (2011).

21. Cui, P. et al. Nonvolatile memory device using gold nanoparticles covalently bound to reduced graphene oxide. Acs Nano 5, 6826-6833 (2011).

22. Doh, Y. J. \& Yi, G. C. Nonvolatile memory devices based on few-layer graphene films. Nanotechnology 21, 105204 (2010).

23. Wang, H. M., Wu, Y. H., Cong, C. X., Shang, J. Z. \& Yu, T. Hysteresis of electronic transport in graphene transistors. Acs Nano 4, 7221-7228 (2010).

24. Lee, Y. G. et al. Fast transient charging at the graphene/SiO2 interface causing hysteretic device characteristics. Appl. Phys. Lett. 98, 183508 (2011).

25. Bertolazzi, S., Brivio, J. \& Kis, A. Stretching and breaking of ultrathin MoS2. Acs Nano 5, 9703-9709 (2011).

26. Mayorov, A. S. et al. Micrometer-scale ballistic transport in encapsulated graphene at room temperature. Nano Lett. 11, 2396-2399 (2011).

27. Brown, W. D. \& Brewer, J. Nonvolatile Semiconductor Memory Technology: A Comprehensive Guide to Understanding and to Using NVSM Devices (IEEE Press, 1998).

28. Yang, H. et al. Graphene barristor, a triode device with a gate-controlled Schottky barrier. Science 336, 1140-1143 (2012).

29. Sekitani, T. et al. Organic nonvolatile memory transistors for flexible sensor arrays. Science 326, 1516-1519 (2009).

30. Kim, S. J. \& Lee, J. S. Flexible organic transistor memory devices. Nano Lett. 10, 2884-2890 (2010).

31. Schlaf, R., Lang, O., Pettenkofer, C. \& Jaegermann, W. Band lineup of layered semiconductor heterointerfaces prepared by van der Waals epitaxy: Charge transfer correction term for the electron affinity rule. J. Appl. Phys. 85, 2732-2753 (1999)

32. Hughes, H. P. \& Starnberg, H. I. Electron Spectroscopies Applied to LowDimensional Structures (Kluwer Academic Publishers, 2000).

33. Cumings, J. \& Zettl, A. Field emission and current-voltage properties of boron nitride nanotubes. Solid State Commun. 129, 661-664 (2004).

34. Yu, Y. J. et al. Tuning the graphene work function by electric field effect. Nano Lett. 9, 3430-3434 (2009).
35. Li, H., Zhang, Q., Liu, C., Xu, S. H. \& Gao, P. Q. Ambipolar to unipolar conversion in graphene field-effect transistors. ACS Nano 5, 3198-3203 (2011).

36. Imam, S. A. et al. Charge transfer hysteresis in graphene dual-dielectric memory cell structures. Appl. Phys. Lett. 99, 082109 (2011).

37. Castro Neto, A. H., Guinea, F., Peres, N. M. R., Novoselov, K. S. \& Geim, A. K. The electronic properties of graphene. Rev. Mod. Phys. 81, 109-162 (2009).

38. Xu, Y. N. \& Ching, W. Y. Calculation of ground-state and optical-properties of boron nitrides in the hexagonal, cubic, and Wurtzite structures. Phys. Rev. B 44, 7787-7798 (1991).

39. Oneill, A. G. An explanation of the asymmetry in electron and hole tunnel currents through ultra-thin Sio2-films. Solid State Electron 29, 305-310 (1986).

40. Watanabe, K. Dependence of effective carrier lifetime in iron-doped silicon crystals on the carrier injection level. Semicond. Sci. Tech. 11, 1713-1717 (1996).

41. Kong, Y. C. et al. Charge storage characteristics in Al/AIN/Si metal-insulatorsemiconductor structure based on deep traps in AIN layer. Appl. Phys. A 90, 545-548 (2008).

42. Liu, Z. et al. Direct growth of graphene/hexagonal boron nitride stacked layers. Nano Lett. 11, 2032-2037 (2011).

43. Lee, C. et al. Anomalous lattice vibrations of single- and few-layer $\mathrm{MoS}_{2}$. ACS Nano 4, 2695-2700 (2010)

44. Gupta, A. et al. Accurate determination of ultrathin gate oxide thickness and effective polysilicon doping of CMOS devices. IEEE Electron Dev. Lett. 18, 580-582 (1997).

\section{Acknowledgements}

This research was supported by the Basic Science Research Program through the National Research Foundation of Korea (NRF), funded by the Ministry of Education, Science and Technology (2009-0083540, 2011-0010274), and by the U.S. National Science Foundation (DMR-1122594)

\section{Author contributions}

M.S.C., G.H.L. and Y.J.Y. designed the research project and supervised the experiment. M.S.C., G.H.L. and Y.J.Y. performed device fabrication under the supervision of J.H. and P.K. G.H.L. performed optical spectroscopy and data analysis. M.S.C., D.Y.L. and S.H.L performed measurements of devices under the supervision of W.J.Y.'s supervision. M.S.C., G.H.L., and W.J.Y. analyzed the data and wrote the paper.

\section{Additional information}

Supplementary Information accompanies this paper at http://www.nature.com/ naturecommunications

Competing financial interests: The authors declare no competing financial interests.

Reprints and permission information is available online at http://npg.nature.com/ reprintsandpermissions/

How to cite this article: Choi, M. S. et al. Controlled charge trapping by molybdenum disulphide and graphene in ultrathin heterostructured memory devices. Nat. Commun. 4:1624 doi: 10.1038/ncomms2652 (2013). 